\title{
Component resolved diagnosis of walnut allergy in young children: Jug $r 1$ as a major walnut allergen
}

\author{
Jeongmin Lee, ${ }^{\star}$ Kyunguk Jeong, Se-ah Jeon, Sooyoung Lee
}

\begin{abstract}
Background: Walnuts (WN) are one of the main causes of tree nut allergies. However, the potential value of component resolved diagnosis (CRD) for WN allergy is controversial.

Objective: We analyzed the clinical and immunological features of clinical WN allergy and the usefulness of CRD in young children.

Methods: Forty-one participants with a history of ingesting WN who were assessed for serum-specific IgE to WN (WN-sIgE) using CRD (ImmunoCAP ISAC 112) at the Department of Pediatrics in Ajou University Hospital were enrolled and their demographic profiles, clinical symptoms, and laboratory findings were evaluated.

Results: A total of 32 patients were diagnosed with clinical WN allergy, of which 31 had specific immunoglobulin E antibodies to Jug $r 1$ (Jug r1-sIgE). The Jug r 1-sIgE levels were higher in WN-allergic patients than in WN-tolerant patients and significantly higher in patients with anaphylaxis than in the WN-tolerant patients. Receiver-operating characteristic curves demonstrated that the Jug $\mathrm{r}$ 1-sIgE level was much better in discriminating between clinical WN allergy and $\mathrm{WN}$ tolerance in young children than the WN-sIgE level.

Conclusions: Jug $\mathrm{r} 1$ is the major component allergen in young children with clinical WN allergy. To measure Jug $\mathrm{r}$ 1-sIgE appears to be a promising approach for both diagnosis and predicting severity in young children with a history of suspected WN allergy.
\end{abstract}

Key words: Jug r 1, walnut, allergy, children, ISAC, component resolved diagnosis

\section{Affiliation:}

Department of Pediatrics, Ajou University School of Medicine, Suwon, Korea

\section{* Current address:}

Department of Pediatrics, Yonsei University, Wonju College of Medicine, Wonju, Korea

\section{Introduction}

Walnut (WN) is one of the many allergens responsible for cases of tree nut (TN) allergy and can induce fatal allergic reactions. ${ }^{1-3}$ Based on a Korean survey performed in 2008, no individual was documented to be allergic to $\mathrm{WN} .{ }^{4}$ However, WN imports have quadrupled in the last 10 years, ${ }^{5}$ and $\mathrm{WN}$ now ranks as the third most common food that induces anaphylaxis in Korean children. ${ }^{3,6}$ Nevertheless, there has been minimal study on WN allergy in very young children to date. $^{6-9}$

A double-blinded, placebo-controlled food challenge (DBPCFC) is the gold standard to confirm food allergies but is contraindicated in patients with a previous history of

\section{Corresponding author:}

Sooyoung Lee

Department of Pediatrics, Ajou University School of Medicine, 164

Worldcup-ro, Yeongtong-gu 16499, Suwon, Korea

E-mail: jsjs87@ajou.ac.kr

anaphylaxis. In fact, $\mathrm{WN}$ often induces severe allergic reactions that necessitate close supervision by experienced allergists during DBPCFC. In 2008, Maloney et al. ${ }^{10}$ investigated diagnostic decision points (DDPs) for WN, indicating that patients with WN-specific immunoglobulin E (sIgE) levels greater than $18.5 \mathrm{kU} / \mathrm{L}$ have a very high probability of experiencing a reaction to $\mathrm{WN}$.

Recombinant technology now allows for the production of components of WN allergen that can be used for component resolved diagnosis (CRD) ${ }^{11}$ CRD may be useful to identify the most problematic allergens in patients with specific sensitization patterns and could help to distinguish anaphylaxis 
from milder symptoms. ${ }^{7,12,13}$ At present, eight WN allergens have been identified and characterized from English walnut (Juglans regia), ${ }^{14}$ three of which are available in the ImmunoCAP ISAC system (Thermo Fisher Scientific Inc., Uppsala, Sweden): 2 S albumin seed storage protein (Jug r 1; 15-16

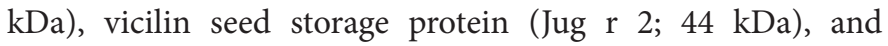
non-specific lipid transfer protein (Jug r 3 or LTP; $6 \mathrm{kDa}$ ). However, the specific allergens involved in the allergic response to $\mathrm{WN}$ were found to differ among patients and were dependent on age, geographic region, and pollinosis. Further, the additional diagnostic value provided by CRD for $\mathrm{WN}$ allergy has proven to be controversial. ${ }^{11-13,15}$

Thus, the aim of this study was to investigate the clinical and immunological characteristics of young Korean children with clinical WN allergy to identify the major allergenic components recognized in this population. Furthermore, we investigated whether additionally performing CRD has greater benefits for diagnosing WN allergy than simply measuring crude WN-sIgE.

\section{Methods \\ Study population}

Based on a retrospective review of medical records, 189 patients aged 12 years or younger who visited the Ajou University Hospital (Suwon, Korea) underwent the ImmunoCAP test (Thermo Fisher Scientific Inc., Uppsala, Sweden) for WNsIgE levels between May 2010 and May 2015. Among these patients, the parents of 41 patients provided informed consent to participate in additional serological analysis, and serum samples were taken simultaneously and stored at $-20^{\circ} \mathrm{C}$.

The diagnostic criteria for clinical WN allergy were as follows: ${ }^{16}$ 1) WN-sIgE $\geq 0.10 \mathrm{kU} / \mathrm{L} ; 2$ ) immediate reaction in the skin, gastrointestinal, or respiratory organs within $2 \mathrm{~h}$ of exposure; and 3) repeated experience or met the diagnostic criteria for anaphylaxis. ${ }^{17}$ Individuals with $\mathrm{WN}$-sIgE $\geq 0.10 \mathrm{kU} / \mathrm{L}$ but who were asymptomatic after consumption were classified as tolerant (T). Moreover, three patients with atopic dermatitis $(\mathrm{AD}), \mathrm{WN}-\mathrm{sIgE}<0.10 \mathrm{kU} / \mathrm{L}$, and who were asymptomatic after exposure were included as the control (C) group. Urticaria was defined as skin erythema including hives, and angioedema was defined as mucosa swelling with or without hives. We excluded cases limited to the perioral area. Controls were screened for those who were diagnosed with AD but not diagnosed with pollinosis.

The study was approved by the institutional review board at Ajou University Medical Center (MED-KSP-12-381).

\section{Microarray}

CRD was performed on all patients using the commercially available immune solid-phase allergen chip (ISAC) immunoassay ImmunoCAP ISAC (CRD 112; Thermo Fisher Scientific Inc., Uppsala, Sweden) according to the manufacturer's guidelines and as reported previously. ${ }^{11}$ Data are expressed as ISAC standardized units (ISU/L, ISU), and the decision threshold was set at 0 .

\section{Statistical analysis}

Statistical analyses were performed using $\mathrm{R}$ version 3.0.2 software ( $\mathrm{R}$ Foundation for Statistical Computing, Vienna, Austria). Normality was tested, and $P$-values for continuous variables were calculated using the Kruskall-Wallis test. Fisher's exact test was performed to compare categorical variables. Pearson correlation analysis was performed to analyze the correlation between WN-sIgE (kU/L) and Jug r 1-sIgE (ISU) concentrations. The best cutoff was found using receiver operation characteristic (ROC) curve analysis, and area under curve values (AUCs) were compared using the Delong test. $P$ $<0.05$ was considered statistically significant.

\section{Results}

\section{Demographic and clinical characteristics of patients}

Among the 41 patients, 32 had WN-sIgE $\geq 0.10 \mathrm{kU} / \mathrm{L}$ $(0.10-91.3 \mathrm{kU} / \mathrm{L})$ and met the clinical diagnostic criteria for WN allergy. The median age was 28 months, with a range of 5-108 months. Symptoms from exposure included anaphylaxis (Ana, $n=7$ ), angioedema (Ang, $n=10$ ), and urticaria ( $U$, $\mathrm{n}=15)$. Among the 7 patients with Ana, all patients showed skin rash and respiratory symptoms, and none of the patients had digestive, circulatory, or neurologic symptoms. Two patients had other food allergies (pine nut and peanut, respectively). Among the 10 patients with Ang, the youngest was 8 months old, and developed hives and edema after consuming ground walnut mixed in water. Ang patients were significantly younger than the other symptom groups $(\mathrm{p}<0.05)$. Among the 12 patients diagnosed with $\mathrm{U}$, the youngest patient was a 5-month-old breast milk-fed infant who had repeated skin rashes with wheals when the mother consumed WN while breastfeeding.

With regard to other allergic comorbidities, asthma was present in 3 Ana patients, 1 Ang patient, and $1 \mathrm{U}$ patient, while allergic rhinitis was present in 4 Ana patients, 2 Ang patients, and $1 \mathrm{U}$ patient. AD was present in 4 Ana patients, 1 Ang patients, and $8 \mathrm{U}$ patients.

Feeding history was taken from 21 patients. Fourteen (66.7\%) patients were exclusively breastfed for the first four months, 7 (50.0\%) of whom experienced an allergic reaction to $\mathrm{WN}$ on their first exposure.

Further, 7 children had WN-sIgE $\geq 0.10 \mathrm{kU} / \mathrm{L}(0.42-53.5$ $\mathrm{kU} / \mathrm{L}$ ) but did not develop symptoms upon exposure $(\mathrm{T})$. The mean age of these children was 55 months, and 6 of those $(85.7 \%)$ had an allergy to other foods. The comorbidity rate for other food allergies was significantly higher among $\mathrm{T}$ patients $(\mathrm{p}<0.05$, Table 1$)$

\section{Immunological characteristics of patients and the additional diagnostic value of Jug $r 1$}

The ImmunoCAP ISAC (CRD 112) assay indicated that 31 of the 32 clinical WN-allergic patients (96.9\%) had specific IgE antibodies to Jug $r 1$ and only 1 (U-9) was positive for Jug r 2-sIgE isolate. The comparison of the immunological features in relation to clinical symptoms showed that the Jug $r$ 1-sIgE concentration varied significantly between the groups ( $\mathrm{p}<0.05)$. It was higher in the Ana (12.9 vs $0.8 ; \mathrm{p}<0.05)$, Ang (5.8 vs $0.8 ; \mathrm{p}=0.48)$, and $\mathrm{U}(6.4$ vs $0.8 ; \mathrm{p}=0.60$ ) group 
Table 1. Comparison of patient characteristics among groups.

\begin{tabular}{|c|c|c|c|c|c|}
\hline Group & $\begin{array}{l}\text { Anaphylaxis } \\
\qquad(\mathrm{n}=7)\end{array}$ & $\begin{array}{l}\text { Angioedema } \\
\qquad(\mathbf{n}=10)\end{array}$ & $\begin{array}{l}\text { Urticaria } \\
(\mathrm{n}=15)\end{array}$ & $\begin{array}{l}\text { Tolerant } \\
(\mathrm{n}=7)\end{array}$ & $P$ \\
\hline $\begin{array}{l}\text { Age } \\
\text { (months; median, range) }\end{array}$ & $\begin{array}{c}41 \\
(15,108)\end{array}$ & $\begin{array}{c}20.5 \\
(8,32)\end{array}$ & $\begin{array}{c}32 \\
(5,58)\end{array}$ & $\begin{array}{c}55 \\
(16,96)\end{array}$ & 0.020 \\
\hline \multicolumn{6}{|l|}{ Sex } \\
\hline Female & $2(28.6 \%)$ & $5(50.0 \%)$ & $8(53.3 \%)$ & $0(0.0 \%)$ & 0.069 \\
\hline Male & $5(71.4 \%)$ & $5(50.0 \%)$ & $7(46.7 \%)$ & 7 (100.0\%) & \\
\hline \multicolumn{6}{|l|}{ Comorbidity } \\
\hline Other FA & $2(28.6 \%)$ & $0(0.0 \%)$ & $6(40.0 \%)$ & $6(85.7 \%)$ & 0.002 \\
\hline Asthma & $3(42.9 \%)$ & $1(10.0 \%)$ & $1(6.7 \%)$ & $2(28.6 \%)$ & 0.119 \\
\hline Atopic dermatitis & $4(57.1 \%)$ & $1(10.0 \%)$ & $8(53.3 \%)$ & $3(42.9 \%)$ & 0.118 \\
\hline Allergic rhinitis & $4(57.1 \%)$ & $2(20.0 \%)$ & $1(6.7 \%)$ & $2(2.0 \%)$ & 0.059 \\
\hline $\begin{array}{l}\text { Jug r1-sIgE } \\
\text { (ISU, median; IQR) }\end{array}$ & $\begin{array}{c}12.9 \\
(11.8 ; 19.4)\end{array}$ & $\begin{array}{c}5.8 \\
(3.0 ; 9.5)\end{array}$ & $\begin{array}{c}6.4 \\
(1.8 ; 13.3)\end{array}$ & $\begin{array}{c}0.8 \\
(0.0 ; 3.9)\end{array}$ & 0.020 \\
\hline $\begin{array}{l}\text { Jug r1-sIgE/WN-sIgE } \\
\text { (median; IQR) }\end{array}$ & $\begin{array}{c}1.5 \\
(0.9 ; 2.7)\end{array}$ & $\begin{array}{c}1.0 \\
(0.3 ; 2.2)\end{array}$ & $\begin{array}{c}0.8 \\
(0.4 ; 2.8)\end{array}$ & $\begin{array}{c}0.1 \\
(0.0 ; 0.7)\end{array}$ & 0.090 \\
\hline $\begin{array}{l}\text { WN-sIgE } \\
\text { (kU/L, median; IQR) }\end{array}$ & $\begin{array}{c}8.4 \\
(4.5 ; 20.2)\end{array}$ & $\begin{array}{c}11.9 \\
(2.0 ; 27.4)\end{array}$ & $\begin{array}{c}7.3 \\
(1.0 ; 18.8)\end{array}$ & $\begin{array}{c}5.8 \\
(4.0 ; 7.1)\end{array}$ & 0.640 \\
\hline
\end{tabular}

The $p$-values $(p)$ for continuous variables were calculated by Kruskall-Wallis (post hoc) test and those for categorical variables were calculated by Fisher's exact test; $p$-values $<0.05$ were considered statistically significant.

FA, food allergy; WN-sIgE, walnut-specific immunoglobulin E on ImmunoCAP; Jug r 1-sIgE, Jug r 1-specific immunoglobulin E on ImmunoCAP ISAC (CRD 112); IQR, interquartile range

(a) Jug r 1-slgE (ISU)

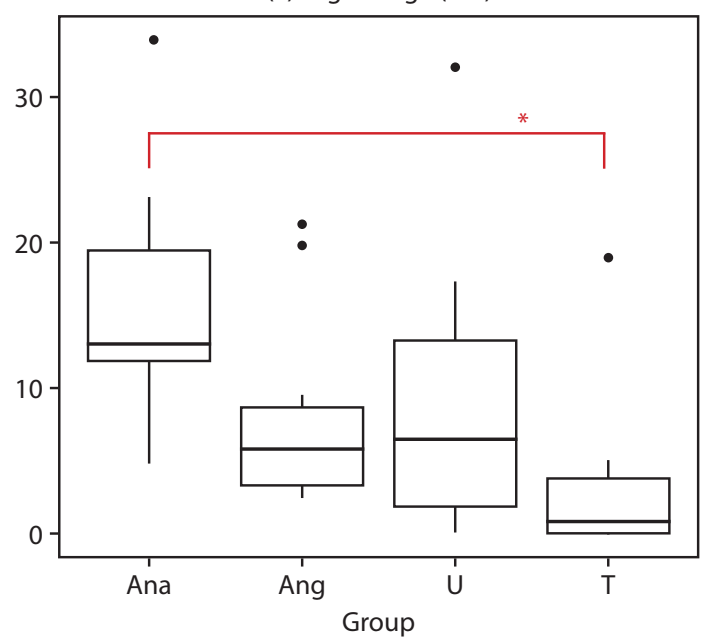

(b) WN-slgE (kU/L)

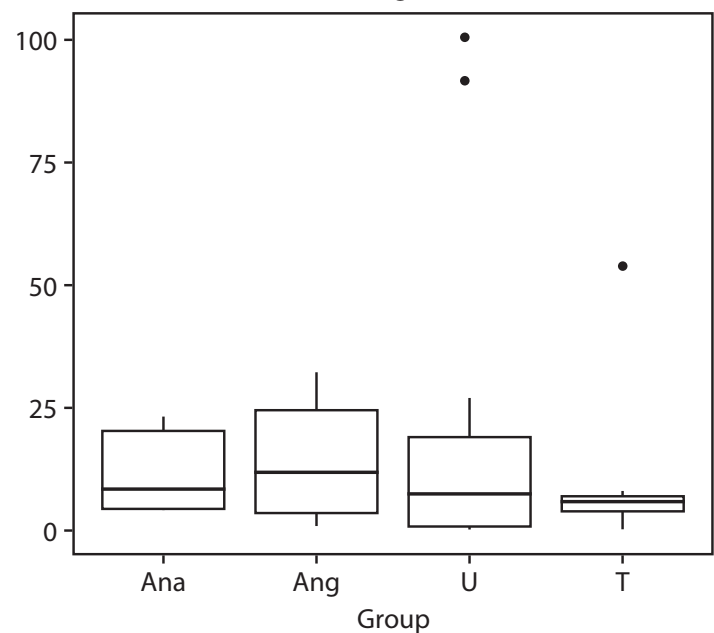

Figure 1. Levels of specific IgE to (a) Jug r 1, and (b) walnut according to the clinical history of anaphylaxis (Ana), angioedema (Ang), urticaria (U), and tolerance $(\mathrm{T}) .{ }^{\star} p<0.05$

than in the T group (Figure 1, Table 1). In contrast, no statistically significant differences in the WN-sIgE concentration were detected among those groups. In addition, the WN-sIgE concentration of 23 out of $32(71.9 \%)$ clinical $\mathrm{WN}$-allergic children was below DDP level $(\leq 18.5 \mathrm{kU} / \mathrm{L})$.

We further analyzed the ImmunoCAP ISAC results for legumes and TNs in 32 clinically $\mathrm{WN}$-allergic patients. All patients were sensitized to the storage protein of $\mathrm{WN}$, and none was exclusively sensitized to the LTP antigen, Jug $r$ 3. Fifteen patients $(46.9 \%)$ were co-sensitized to more than one storage protein. The protein with the highest positivity was the soy storage protein Gly $\mathrm{m} 6$ ( $\mathrm{n}=8,25.0 \%)$, followed by hazel (Cor a 9, $\mathrm{n}=7,21.9 \%$ ) and peanut (Ara h 2, n =6, 18.8\%; Figure 2). Only two patients (37 months and 66 months old) were co-sensitized to PR-10, and both had a history of pollen food syndrome. Cross-reactive carbohydrates (CCD) were negative in all patients. Meanwhile, six patients in the $\mathrm{T}$ group $(85.7 \%)$ were co-sensitized to more than one storage protein. Information on clinical hypersensitivity to other TNs was not fully available for all patients. 


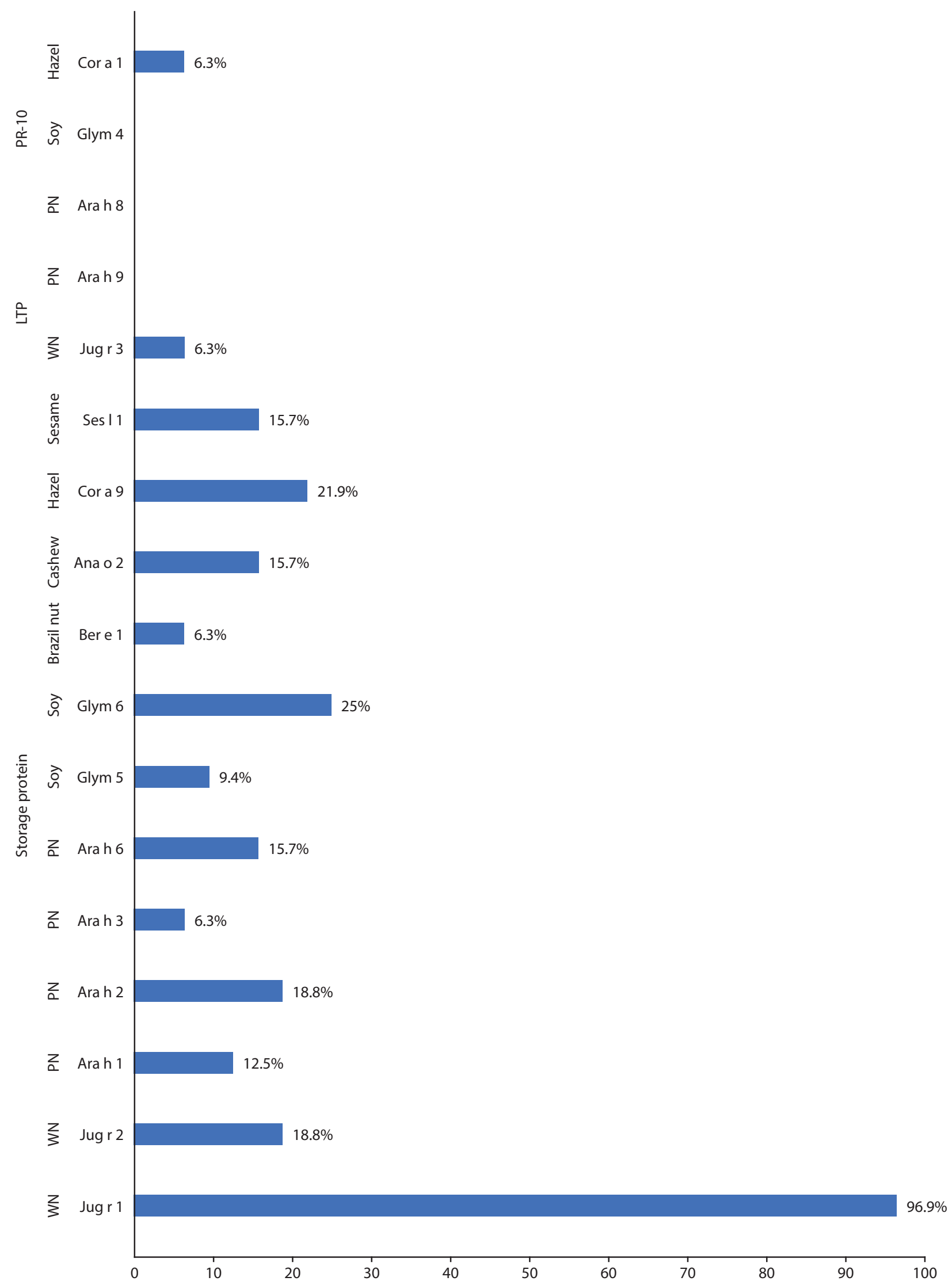

Figure 2. IgE to peanut, soy, and tree nuts detected in clinically walnut allergic children $(\mathrm{n}=32)$ determined by ImmunoCAP ISAC (CRD 112). 


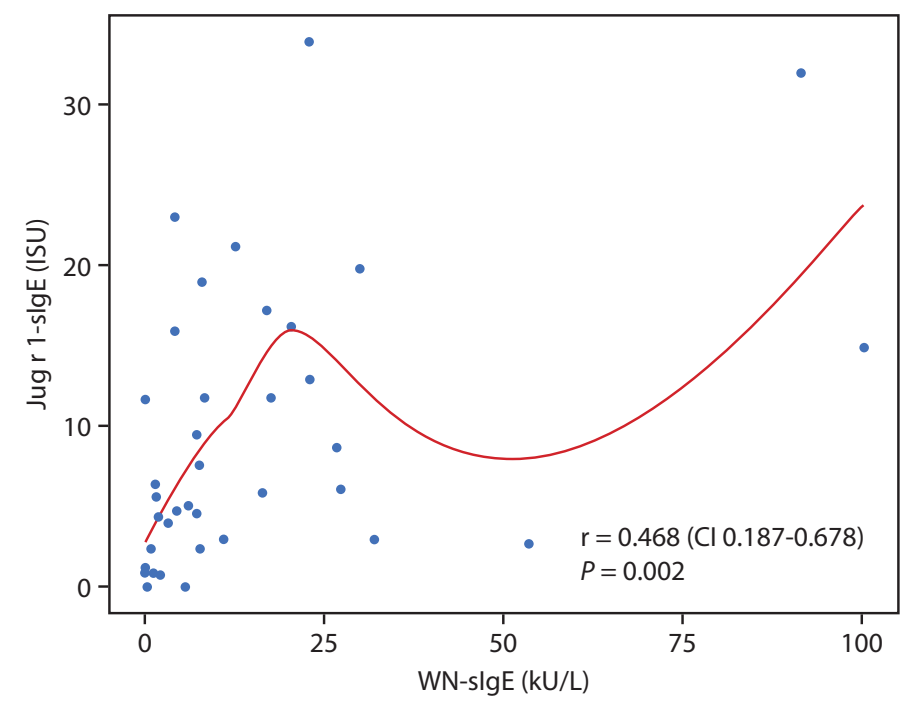

Figure 3. Correlation between walnut-specific IgE measured on ImmunoCAP, and Jug r 1-specific IgE measured on ImmunoCAP ISAC $(\mathbf{n}=39)$.

Jug r 1-sIgE, Jug r 1-specific immunoglobulin E (ISU); WN-sIgE, walnut-specific immunoglobulin E (kU/L); CI, confidence interval.

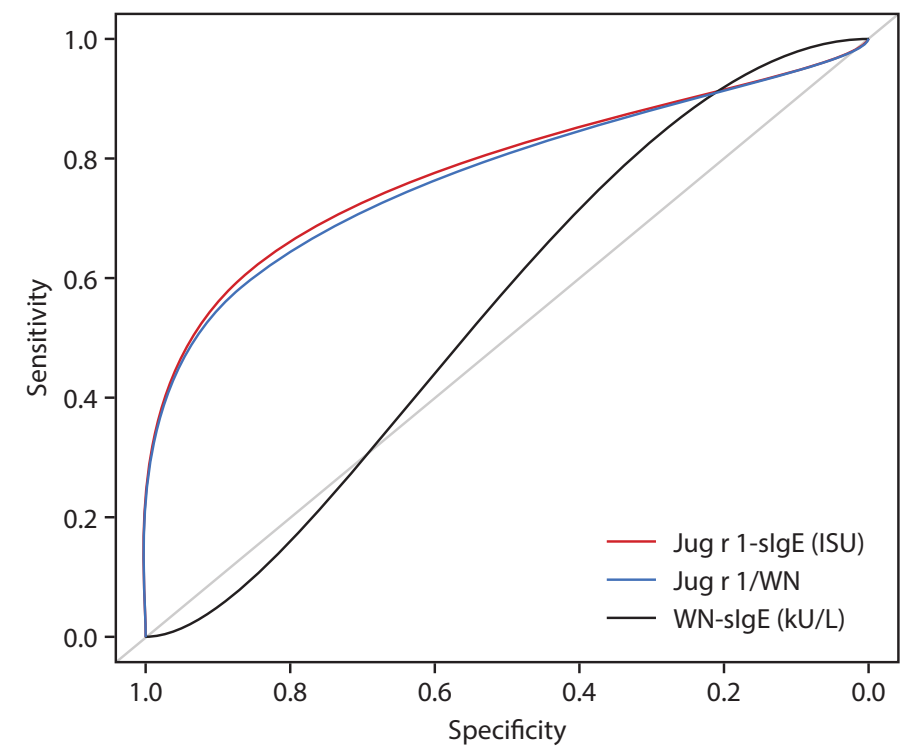

\begin{tabular}{lccccccc} 
Components & Best cut off & Sensitivity (\%) & Specificity (\%) & PPV (\%) & NPV (\%) & AUCs & p-value \\
\hline WN-sIgE (kU/L) & 5.96 & 85.71 & 46.88 & 6.2 & 73.9 & 0.563 & 0.592 \\
\hline Jug r 1-sIgE (ISU) & 4.21 & 57.14 & 96.87 & 8.8 & 20.0 & 0.788 & 0.017 \\
Jug r 1/WN & 0.28 & 57.14 & 93.75 & 9.1 & 33.3 & 0.781 & 0.013 \\
\hline
\end{tabular}

Figure 4. Receiver-operating characteristic curves of IgE specific to Jug r 1 and ratio of Jug r 1/walnut-specific IgE compared with the specific IgE to crude walnut extract with regard to the ability to discriminate between an allergy and tolerance to walnut. AUCs, area under the curve values. 
A positive, significant correlation between Jug $\mathrm{r}$ 1-sIgE and WN-sIgE levels was found ( $\mathrm{r}=0.468$; CI 0.187-0.678, $\mathrm{p}<$ 0.05 ; Figure 3 ). The ROC curves showed that the Jug r 1-sIgE measurement was better than the WN-sIgE measurement in discriminating between clinical $\mathrm{WN}$ allergy and $\mathrm{WN}$ tolerance in young children $(\mathrm{AUC}=0.788, \mathrm{p}<0.05$; Figure 4$)$.

\section{Discussion}

To our knowledge, this is the first study to investigate the value of $\mathrm{CRD}$ for $\mathrm{WN}$ allergy in very young children. Although oral food challenge (OFC) testing remains vital for a definite diagnosis, parents often disagree with OFC using nuts in very young children. Consequently, definitive diagnosis is often delayed, which hinders research progress. However, since we have occasionally treated clinically $\mathrm{WN}$-allergic patients, we aimed to explore the benefit of CRD among such young patients for whom the OFC is practically difficult. Furthermore, we examined co-sensitization profiles of other nuts prior to ingestion.

To date, there have been three studies on CRD for WN allergy in older children., ${ }^{7,13}$ In 2014, a study was conducted on 45 children (mean age: 8 years) in Northern Italy, ${ }^{13}$ showing a higher percentage of Ana patients who were positive for Jug r 1-sIgE and Jug r 2-sIgE, when compared with those with mild-moderate WN allergy. In our study, only the Jug r 1-sIgE level was higher in $\mathrm{WN}$-allergic patients than in tolerant patients and this level was significantly higher among patients with anaphylaxis than among patients with WN tolerance, which suggested an association with severity. Our observation that patients with anaphylaxis had higher Jug $\mathrm{r}$ 1-sIgE levels may be explained by the fact that it is specific to a storage protein that contributes to the severe systemic response. However, considering the finding of earlier research that Jug r 1-sIgE level was not associated with the severity of allergic reactions, there is a need to examine whether our observation is specific to the age group examined in this study.

In a 2016 British study, ${ }^{12}$ although the Jug r 1-sIgE level was significantly higher than that among tolerant children, the AUC value was similar to that for WN-sIgE. A recent Japanese study ${ }^{6}$ administered an OFC to 108 children (median age of 6-7 years) and found higher WN-sIgE and Jug r 1-sIgE concentrations among the WN allergy group than among the tolerant group. In contrast to the British and Japanese studies, the WN-sIgE concentrations in our study did not discriminate across groups, and the AUC of WN-sIgE was far weaker than that of Jug $\mathrm{r} 1$-sIgE. This difference may be attributable to the fact that the clinical and immunological features of food allergy depend on age and region. Furthermore, in this study, when the DDP of WN-sIgE was set as $18.5 \mathrm{kU} / \mathrm{L}, 71.9 \%$ of clinically allergic patients were falsely predicted as tolerant; thus, clinicians must be aware of this. ImmunoCAP-ISAC is expensive and limited to research purposes in many countries, but the cost of selecting and implementing Jug $r$ 1-sIgE is similar to that of using WN-sIgE. Hence, based on our results, among the components of WN, to measure Jug r 1-sIgE for diagnosis would be beneficial and cost-effective in this age group.
It is also noteworthy to consider the history of patients who experienced WN allergies on their first exposure. Based on the experience of a patient (Ang-2) who never ingested $\mathrm{WN}$ and did not have $\mathrm{AD}$ but had a $\mathrm{WN}$-allergic reaction at 8 months of age with a WN-sIgE level of $30.0 \mathrm{kU} / \mathrm{L}$. In addition, we found that approximately $50 \%$ of the patients with clinical WN allergy were already poly-sensitized to numerous allergens other than $\mathrm{WN}$, and the majority of them had no previous history of ingestion. We considered that allergy prevention by early introduction might not only be necessary for moderate-to-severe AD patients and the factors affecting individual variations must be studied further.

The $\mathrm{T}$ group showed a high rate of other food allergies and had a significantly lower Jug $\mathrm{r}$ 1-sIgE concentration. Therefore, WN and wide-ranging $\mathrm{TN}$ avoidance guidance must not be given to young children with positive results of serum study only, especially, when their WN-sIgE level is $\geq$ $0.10 \mathrm{kU} / \mathrm{L}$ with a low Jug $\mathrm{r}$ 1-sIgE concentration, and they have already been diagnosed with a soybean or peanut allergy. For the purpose of screening sensitization to the other tree nuts, multiplex component-based allergen microarray would be particularly useful for infants with moderate to severe $A D$, for whom skin tests are practically difficult. Many of our patients were breastfed or on a mixed-fed diet, which may have contributed to the sensitization, but transcutaneous sensitization through $\mathrm{AD}$ as well as co-sensitization due to cross-antigenicity are also possible. ${ }^{18-20}$ Additional studies are needed to provide more accurate diet guidance and, despite practical challenges, a definitive diagnosis based on an OFC appears to be crucial before providing avoidance.

This study has some limitations that should be discussed. The major limitation of this study was its retrospective design. Moreover, the study was based on self-reported history of allergic reactions, and the oral food challenge test was not conducted in most of patients. However, the results of this study are noteworthy, considering that no previous studies have been conducted with CRD for very young children. The median age of our subjects was far lower than that in previous studies, and thus, the underlying diseases were considerably different from those in previous studies, which was reflected in the result of the CRD. Moreover, we recruited participants based on real-world data according to the level of exposure and symptoms, and that heavy exposure tended to occur in one event. In contrast, in other studies, participants were gradually exposed to $\mathrm{WN}$ by OFC.

In conclusion, measurement of Jug $\mathrm{r} 1$-sIgE on very young patients with suspected WN allergy could be beneficial for diagnosis and severity assessment, because these patients have a higher specific IgE level to this component than WN-tolerant patients and, furthermore, anaphylaxis patients have a significantly higher level than the tolerant patients.

\section{Acknowledgements}

We thank Thermo Fisher Scientific Inc. (Uppsala, Sweden) for providing the kits used in component resolved diagnosis of WN allergy. This research received no specific grant from any funding agency. 


\section{Disclosure statement}

The authors declare that the ISAC CRD kit was donated by Thermo Fisher Scientific Inc. (Uppsala, Sweden). There are no other conflicts of interest related to this work.

\section{Author Contributions}

- Conceptualization: Sooyoung Lee

- Data curation: Se-ah Jeon, Jeongmin Lee

- Formal analysis: Jeongmin Lee, Kyunguk Jeong

- Methodology: Sooyoung Lee, Jeongmin Lee

- Validation, Investigation: Se-ah Jeon, Jeongmin Lee

- Writing original draft: Jeongmin Lee

- Review and editing: Kyunguk Jeong, Jeongmin Lee, Sooyoung Lee

\section{References}

1. Le TM, Lindner TM, Pasmans SG, Guikers CL, Van Hoffen E, Bruijnzeel -Koomen CA, et al. Reported food allergy to peanut, tree nuts and fruit: comparison of clinical manifestations, prescription of medication and impact on daily life. Allergy. 2008;63:910-6.

2. McWilliam V, Koplin J, Lodge C, Tang M, Dharmage S, Allen K. The prevalence of tree nut allergy: A systematic review. Curr Allergy Asthma Rep. 2015;15:54.

3. Lee SY, Ahn K, Kim J, Jang GC, Min TK, Yang HJ, et al. Korean Academy of Pediatric Allergy and Respiratory Diseases Food Allergy and Atopic Dermatitis Study Group. A multicenter retrospective case study of anaphylaxis triggers by age in Korean children. Allergy Asthma Immunol Res. 2016;8:535-40.

4. Lee SY, Kim KW, Ahn K, Kim HH, Pyun BY, Park YM, et al. [Consumer's use and satisfaction of allergic food labels]. Pediatr Allergy Respir Dis. 2011;21:294-301. Korean.

5. Korea Custom Service [Internet]. Seoul: The Korea Custom Service; c2013 [cited 2018 Oct 30]. Tree nut import trends in 10 years; [about 9 screens]. Available from: http://www.customs.go.kr/kcshome/main/search_idol.do ?query=\%EA $\% \mathrm{~B} 2 \% \mathrm{AC} \% \mathrm{EA} \% \mathrm{~B} 3 \% \mathrm{BC} \% \mathrm{~EB} \% \mathrm{~A} 5 \% 98 \&$ queryText=\%EA $\%$ B2\%AC\%EA\%B3\%BC\%EB\%A5\%98\&notWord=\&selZone=\&sel Date $=$ ALL \&beginDate $=$ \&endDate $=\&$ date $1=\&$ date $2=\&$ cateCode $=0 \&$ sortSpec $=$ date $\&$ docStart $=11$

6. Jeong K, Lee SY, Ahn K, Kim J, Lee HR, Suh DI, et al. A multicenter study on anaphylaxis caused by peanut, tree nuts, and seeds in children and adolescents. Allergy. 2017;72:507-10.
7. Sato S, Yamamoto M, Yanagida N, Ito K, Ohya Y, Imai T, et al. Jug r 1 sensitization is important in walnut-allergic children and youth. J Allergy Clin Immunol Pract. 2017;5:1784-6.

8. Lee JM, Kim EJ, Kwon DG, Lee SY. [Clinical characteristics of walnut allergy and evaluation of cross-reactivity between walnut and peanut in children under 4 years of age]. Pediatr Allergy Respir Dis. 2011;21:261-8. Korean.

9. Lee J, Jeong K, Jeon SA, Lee S. Immunoglobulin E-binding proteins of cooked walnuts in Korean children. Allergy Asthma Immunol Res. 2018; 10:363-9.

10. Maloney JM, Rudengren MR, Ahlstedt S, Bock SA, Sampson HA. The use of serum-specific IgE measurements for the diagnosis of peanut, tree nut, and seed allergy. J Allergy Clin Immunol. 2008;122:145-51.

11. Ferrer M, Sanz ML, Sastre J, Cuvillo A, Montoro J, Jauregui I, et al Molecular diagnosis in allergology: application of the microarray technique. J Investig Allergol Clin Immunol. 2009;19:19-24.

12. Mew R, Borres M, Sjölander S, du Toit G. A retrospect study into the utility of allergen components in walnut allergy. Pediatr Allergy Immunol. 2016; 27:750-2.

13. Ciprandi G, Pistorio A, Silvestri M, Rossi GA, Tosca MA. Walnut anaphylaxis: the usefulness of molecular-based allergy diagnostics. Immunol Lett. 2014;161:138-9.

14. Allergen Nomenclature [Internet]. Nebraska: WHO/IUIS Allergen Nomenclature Sub-Committee; c2007-2019 [cited 2017 Nov 30]. Available from: http://allergen.org/search.php?Species=Juglans\%20regia

15. Blankestijn MA, Blom WM, Otten HG, Baumert JL, Taylor SL, Bruijnzeel -Koomen CA, e al. Specific IgE to Jug r 1 has no additional value compared with extract-based testing in diagnosing walnut allergy in adults. Allergy Clin Immunol. 2017;139:688-90.

16. Park M, Kim D, Ahn K, Kim J, Han Y. Prevalence of immediate-type food allergy in early childhood in Seoul. Allergy Asthma Immunol Res. 2014;6:131-6.

17. Simons FE, Ardusso LR, Dimov V, Ebisawa M, El-Gamal YM, Lockey RF, et al. World Allergy Organization. World Allergy Organization Anaphylaxis Guidelines: 2013 update of the evidence base. Int Arch Allergy Immunol. 2013;162:193-204.

18. de Leon MP, Glaspole IN, Drew AC, Rolland JM, O’Hehir RE, Suphioglu C. Immunological analysis of allergenic cross-reactivity between peanut and tree nuts. Clin Exp Allergy. 2003;33:1273-80.

19. Kulis M, Pons L, Burks AW. In vivo and T cell cross-reactivity between walnut, cashew and peanut. Int Arch Allergy Immunol. 2009;148:109-17.

20. Clark AT, Ewan PW. The development and progression of allergy to multiple nuts at different ages. Pediatr Allergy Immunol. 2005;16:507-11. 